\title{
The Quantitative Forecast of Precipitation with the Numerical Prediction Method*
}

\author{
By Staff Members in Tokyo University** \\ Geophysical Institute, Tokyo University \\ (Manuscript received 5 February 1955, revised 18 August 1955)
}

\begin{abstract}
We made a quantitative forecast of precipitation based on the numerical prediction method, using three layer model of the atmosphere. Treating the transport of water vapour three dimensionally and considering the temperature change, the excess of water vapour over saturation was estimated, which we assumed to fall off entirely as precipitation.

These calculations were performed by the graphical method designed by Fj $\phi \mathrm{rtoft}$, using the geostrophic approximation for the wind velocity.

The North American Continent was chosen as the forecasting area. For 12 hour forecast, the coincidence between the observed and forecast precipitation was found to be fairly good both in amount and area. This result suggests the possibility of forecasting weather based on the large scale motion of the air.

Lastly, brief discussions were made on the non-adiabatic effect, which we disregarded in our practical forecasting.
\end{abstract}

\section{\$ 1. Introduction}

In these years the technique of the numerical weather prediction method has remarkably progressed, and the 24 hourly forecast of height pattern on isobaric surface has become possible with a considerable accuracy. Furthermore, introduction of this method into routine work has already been realized in Joint Numerical Weather Prediction Unit in U.S.A. by the aid of high speed computing machine. The forecast quantity, however, has mostly been confined only to the change in the height pattern. It is true, indeed, that the change in the height pattern is a fundamental and important element for the weather forecast, but the numerical prediction of precipitation or cloud distribution is also an utterly urgent problem.

Two methods have been proposed to attack this problem: the one is to estimate upward current and the other is to consider transport of water vapour. In the former category, Fliegle [11], Miller [15], Bellamy [3] and Panofsky [16] designed the method of calculation of divergence, using the observation data of temperature and the observed wind data. Along this line of attack, Arizumi [1] and Masuda [14] also proposed the similar

* Division of Meteorology, Contribution No. 84.

* M. Komabayasi, K. Miyakoda, M. Aihara, S. Manabe and K. Katow. method. Later on, Miller, Syōno [18], Kasahara and Panofsky emphasized the vertical current in the lower level of the atmosphere effective for precipitation, and they devised the method of calculation of upward current induced by vorticity making use of the principle of Ekman spiral. Eliassen and Hubert [9] computed the large scale vertical motion from the vorticity equation at several levels and compared it with the observed distribution of precipitation and cloudiness. Collins and Kuhn [8] also treated the problem in the same manner as Eliassen and Hubert. In recent years, several researches have also been made from the point of view of numerical prediction. The merit of this standpoint lies in the forecast of vertical current in the successive calculation with respect to time. Phillips [17], Charney and Phillips [7] compared the pattern of upward current thus calculated based on the two layer model of the atmosphere with the observed weather.

On the other hand, quantitative investigation of precipitation has been made by many authors taking the water vapour into consideration. For instance, Asakura [2] treated the continuity equation for water vapour and tested the validity of the equation in the actual atmosphere. Thompson and Collins [20] calculated the intensity of precipitation by using 
Fulks' formula with some modifications to compensate for unsaturated initial conditions. Kuhn [12] calculated the amount of precipitation for 12 hours based on the similar principle. Spar [18] also made quantitative forecast of precipitation by estimating the amount of supersaturation, based on the assumption that the temperature did not change.

In our forecast, however, we made an attempt to introduce not only the transport of water vapour, but also the time change in temperature $^{1)}$. In order to predict the temperature, it is necessary to solve the simultaneous equations of Poisson type governing the behaviour of temperature field. Having no high speed computing machine at present, we used a graphical method for solving these equations, which was developed by Fjortoft [10].

However, there were two problems to be ascertained before the actual forecasting of precipitation was performed: the one is the possibility to estimate rainfall quantitatively from large scale motion of the air, and the other is whether radiosonde data of humidity have a sufficient accuracy to be used in the quantitative forecast of rainfall. As a preliminary test to clarify these two points, we calculated the amount of precipitation by continuity relation of water vapour and compared it with the observation. In this test, the values of humidity were read from the radiosonde data on five standard levels, and water vapour was assumed to be transported by large scale three dimensional motion of the air. As the result of this preliminary test, it was tentatively concluded that, with radiosonde data of humidity by existing frame of network, quantitative forecasting of precipitation is possible to a considerable extent.

\section{§2. Method of Precipitation Forecast}

The forecasting method of precipitation we used is based on the following assumption; the excess of water vapour over saturation is entirely equal to the amount of precipitation,

1) According to the abstract presented in the 133rd National Meeting of the American Meteorological Society, J. Smagorinsky and G. O. Collins have recently made the nearly same forecast with ours and obtained good results in the precipitation forecast comparable to the predicted flow. i.e.; we ignore both the storage of water droplets in clouds and evaporation of cloud droplets and raindrops. First, we estinnate the transport of water vapour without change of mass and then we compare the quantity thus estimated with the saturation vapour pressure which calculated from the forecast temperature. If the former is larger than the latter, the excess should condense to water drops and fall down as rain. Otherwise no precipitation takes place. Thus, the saturation vapour pressure and the movement of water vapour are the fundamental elements to be forecast. The former quantity is to be determined by the temperature of the atmosphere, and the latter by the horizontal and vertical velocity advection. Now we shall describe the technique how to forecast temperature and specific humidity, and how to calculate vertical current.

\section{a) Forecasting of Temperature and Upward Current}

The temperature should be forecast considering three dimensional advection of the air. Because, it is the adiabatic cooling due to upward motion that causes an increase in relative humidity of an air, while the horizontal advection of the air on isobaric surface does not contribute to the change in relative humidity. This means that the advective method is not applicable in this case and instead the solving the simultaneous equations governing the three dimensional behaviour of the atmosphere is required, though it is complicated. The original form of the forecasting scheme along this line was first presented by Charney and Phillips. Hereafter we shall outline this scheme for the convenience of the following discussions, but in a slightly modified form.

In practice, we forecast primarily the thickness change $\frac{\partial h}{\partial t}$, which is easily translated into the corresponding temperature change $\frac{\partial T}{\partial t}$, where $h$ and $T$ are the thickness and the mean temperature of the layer between two constant pressure levels $p$ and $p+\delta p$, respectively.

\section{Calculation of thickness change}

As is conventional, the change of absolute vorticity $\frac{\partial \eta}{\partial t}$ is given by the following equa- 
tion, neglecting the term $\omega \frac{\partial \eta}{\partial p}$,

$$
\frac{D \eta}{D t}=f \frac{\partial \omega}{\partial p}
$$

where $\omega$ is identical with $\frac{d p}{d t}$ which corresponds to the vertical velocity in $x \cdot y \cdot p$ coordinate system, or the vertical-p-velocity, and $\frac{D}{D t}=\frac{\partial}{\partial t}+\boldsymbol{V} \cdot \boldsymbol{\nabla}, \boldsymbol{V}$ and $\nabla$ being the horizontal wind velocity and the horizontal differentiation operator, respectively. On the other hand, we assume that the potential temperature of air parcel is conserved ${ }^{2)}$. Then, the conservation law of potential temperature may be written as follows,

$$
d \ln \theta=0
$$

where $\theta$ is the potential temperature and $\frac{d}{d t}=\frac{D}{D t}+\omega \frac{\partial}{\partial p}$. The above equation (2.2) may readily be transformed into

$$
\omega=-\frac{1}{\frac{\partial \ln \theta}{\partial p}} \frac{D \ln \theta}{D t}=\kappa \delta p \frac{D \ln \theta}{D t},
$$

where $\kappa=\left(-\frac{\partial \ln \theta}{\partial p} \cdot \delta p\right)^{-1}$ is the vertical static stability of the atmosphere. Eliminating $\omega$ from these two equations (2.1) and (2.3), using the relation $\delta(\ln \theta)=\delta(\ln h)$ derived from the definition of potential temperature and the equation of state $p=\rho R T$ ( $\rho$ : the density of the air, $R$ : the gas constant of the dry air) and applying the geostrophic approximation to the absolute vorticity $\eta\left(\eta=(1 / f) \nabla^{2} \psi+f, f\right.$ and $\psi$ being Coriolis parameter and the geopotential height of the constant pressure surface), we obtain

$$
\frac{D}{D t}\left[\frac{1}{f} \nabla^{2} \psi+f\right]=\frac{\partial}{\partial p}\left[\kappa \delta p f \frac{D \ln h}{D t}\right] .
$$

Or if we consider that $h=-\frac{\partial \psi}{\partial p} \cdot \delta p$ and introduce into equation (2.4) the linearized absolute potential vorticity $\xi$ which is of the form

$$
\xi=\frac{1}{f}\left[\nabla^{2}+\frac{\partial}{\partial p}\left(\mu \frac{\partial}{\partial p}\right)\right] \psi+f,
$$

the equation (2.4) leads to

2) As for this assumption some discussions are made later.

$$
\frac{D \xi}{D t}=0
$$

where

$$
\mu=\frac{\kappa(\delta p)^{2} \cdot f^{2}}{\bar{h}}
$$

$\bar{h}$ being the standard thickness, to be obtained from the standard atmosphere.

The above equation (2.6) represents the socalled conservation law for absolute potential vorticity. Now we differentiate the equation (2.6) with respect to $p$, and obtain the following form:

$$
\frac{D \tau}{D t}=\frac{\partial \boldsymbol{V}}{\partial p} \delta p \cdot \nabla \xi
$$

where

$$
\tau=\frac{\partial \xi}{\partial p} \delta p=\frac{1}{f}\left[\nabla^{2}+\frac{\partial^{2}}{\partial p^{2}} \mu\right] h .
$$

Equation (2.8) provides us with the thickness tendency. In order to obtain $h$ at the time $\Delta t$ later, we rewrite (2.8) in the following form

$$
\frac{\partial \tau}{\partial t}=-\boldsymbol{V} \cdot \nabla \tau+\frac{\partial \boldsymbol{V}}{\partial p} \delta p \cdot \nabla \xi
$$

and inserting initial values to the right hand side of equation (2.10) we obtain $\frac{\partial \tau}{\partial t}$. Secondly, we apply three-dimensional relaxation calculation on $\frac{\partial \tau}{\partial t}$ based on the relation (2.9) and obtain $\frac{\partial h}{\partial t}$.

Then, making use of the usual finite difference approximation, $h(\Delta t)$ is obtained by the relation $h(\Delta t)=h(0)+\frac{\partial h}{\partial t} \Delta t$, where $h(\Delta t)$ and $h(0)$ are the thicknesses at $\Delta t$ later and at the initial moment, respectively.

It is also added here to obtain the vertical$p$-velocity $\omega$. Equation (2.3) is transformed to by using the relation $\delta(\ln \theta)=\delta(\ln h)$

$$
\omega=\frac{\mu}{\delta p \cdot f^{2}} \frac{D h}{D t} .
$$

In this equation, there are two variable quantities: $\mu$ and $\frac{D h}{D t}$. Though we employ the constant values of $\mu$ in the calculation of $\frac{\partial h}{\partial t}$, introduction of the horizontal variation of $\mu$ was found to give better result in calculating $\omega$. The reason is that in the former case, 
the consideration of the variation of $\mu$ leads to complicated calculation and besides it sometimes causes the computational instability, while in the latter there is no such apprehension and instead the variance of $\mu$ provides us with a more detailed pattern of $\omega$.

It is worth while to note here that the value $\mu$ is to be obtained from the second derivative of isobaric height with respect to $p$, accordingly the calculation is liable to give considerable errors. In the first attempt to obtain the horizontal distribution of $\mu$, we calculated the values $\mu$ directly from the radiosonde data reported from the respective stations and plotted the calculated values on maps. But the result showed that this attempt was in a complete failure. In the horizontal distribution of $\mu$ thus calculated, there were peaks of magnitude here and there and besides the distribution did not show the sequence with respect to time. Therefore, in the revised method, we calculated its horizontal distribution from the thickness pattern of two layers appropriately smoothed up, and could obtain a seemingly reasonable pattern of $\mu$ from which the insignificant noises and errors would probably have been removed, and which showed a clear sequence different from the one directly calculated from radiosonde data.

Translation of thickness change into temperature change

Here we shall describe brielly the method to estimate temperature from thickness. It is well known that the thickness of a layer is related to its mean temperature $\bar{T}$ through hydrostatic equation. Integrating this equation with respect to $p$, we obtain

$$
\bar{T}=\frac{1}{R} \ln \left(1+\frac{\delta p}{p}\right)(1+0.000608 q)-273.2
$$$$
\text { (in }{ }^{\circ} \mathrm{C} \text { ) }
$$

where $q$ is the specific humidity in $\mathrm{g} / \mathrm{kg}$. In practice, in order to avoid large errors of forecast temperature we do not estimate it directly from the forecast whole thickness, but we calculate the temperature change $\frac{\partial \bar{T}}{\partial t}$ from the forecast thickness change $\frac{\partial h}{\partial t}$ and add it to the initial mean temperature of the layer we are concerned with.

b) Forecasting of the Water Vapour Distribution

First, we shall specify notations of the quantities concerning water vapour. $q$ is the actual specific humidity, $q^{\prime}$ the virtual specific humidity which would be obtained by the advection under the assumption that neither condensation nor evaporation takes place, and $q^{*}$ the saturation specific humidity. As mentioned before, in our forecast process we first compute the virtual specific humidity $q^{\prime}(\Delta t)$ at a time $\Delta t$ later. In order to obtain it, we apply the continuity relation for the specific humidity, i.e.,

$$
\frac{d q}{d t}=0
$$

From this equation the change $\frac{\partial q}{\partial t}$ is obtained as follows

$$
\frac{\partial q}{\partial t}=-\boldsymbol{V} \cdot \nabla q-\omega \frac{\partial q}{\partial p} .
$$

Here we use as the vertical-p-velocity $\omega$, the value obtained in equation (2.11). Inserting the initial value into $q$ on the right hand side of the above equation, and adding $\frac{\partial q}{\partial t} \Delta t$ thus obtained to the initial specific humidity $q(0)$, we get the virtual specific humidity $q^{\prime}(\Delta t)$ at a time $\Delta t$ later

$$
q^{\prime}(\Delta t)=q(0)+\frac{\partial q}{\partial t} \cdot \Delta t
$$

\section{c) Estimation of Oversaturation}

From the temperature predicted in a), we can determine the saturation specific humidity $q^{*}(\Delta t)$ at a time $\Delta t$ later. Here we subtract $q^{*}(\Delta t)$ from $q^{\prime}(\Delta t)$. The difference $q^{\prime}-q^{*}$ represents, if positive, the oversaturation quantity of water vapour, in other words, the amount of condensation for the time interval at. On the contrary, if the difference is negative, there occurs no condensation and $q^{\prime}(\Delta t)$ equals to $q(\Delta t)$. Finally, the amount of precipitation is obtained by integrating $q^{\prime}-q^{*}$ from the bottom to the top of the atmos. phere, where the evaporation of cloud droplets and raindrops and the transport of cloud droplets are disregarded.

In practical calculation, we used the three layer model of the atmosphere, dividing the 
atmosphere into three layers ${ }^{3)}$, of $1000-700 \mathrm{mb}$, $700-500 \mathrm{mb}$ and $500-0 \mathrm{mb}$ (Fig. 1). Besides, the advecting current was approximated by the geostrophic wind. In this respect, we were much concerned whether this approxi-

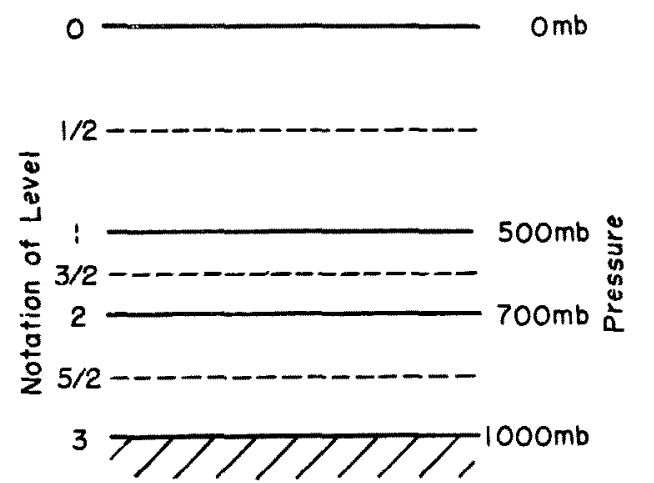

Fig. 1. Schematic illustration for three layer model and notations of levels.

mation might filter away the vertical current effective for the rainfall. For it is well known that the small scale convective current sometimes brings about intense local showers. But we dared to apply the geostrophic approximation to the wind velocity as a first trial of our research.

Furthermore, in the actual computation, having no high speed computer machine yet in Japan, we used Fj $\phi$ rtoft's graphical method taking the mesh size of $600 \mathrm{~km}$ for the calculation of vorticity. So, we did not consider small convective cells smaller than about 2000 $\mathrm{km}$.

As for the time interval, it is usually determined from the computational instability criterion of Courant-Friedrich-Lewy. In our case, however, the upper limit of $\Delta t$ also depends upon the validity of the assumption that the water vapour is conserved during $\Delta t$. In our preliminary test mentioned in $\$ 1$, it was found that $\Delta t$ should be at most 6 hours in the graphical computation. Otherwise, calculated precipitation area shifted forewards too far away from the observed one.

\section{d) Extension of Time Steps}

The above is the outline of the computing method for one step, through which we can

3) The reason why we adopted three layer model will be described later. forecast the temperature and water vapour $\Delta t$ later. However, the quantities thus forecast are, so to speak, the values for the mid-point of the layers. If we want to extend further the time step of calculation, difficulty arises i.e., the informations concerning the water vapour are decreased in number by every time step. For example, in the three layer model of the atmosphere, though the initial informations of water vapour are given on the three standard levels $(1000 \mathrm{mb}, 700 \mathrm{mb}, 500 \mathrm{mb})$, at the end of the first step the levels with information are lessened to two, i.e., $600 \mathrm{mb}$ and 850 $\mathrm{mb}$. This, of course, comes from the computation scheme of specific humidity involving the term $\omega \frac{\partial q}{\partial p}$ in equation (2.14). It is noticed here that with respect to the temperature forecast, such trouble does not occur, for in the computation scheme of $\psi(2.1)$ the term $\omega \frac{\partial \eta}{\partial p}$ is omitted. In the case of the forecast of specific humidity, however, the effect of the vertical transport of water vapour, represented by the term $\omega \frac{\partial q}{\partial p}$, could not be neglected. In this meaning, in order to extend the time step further it is required to forecast the specific humidity on all standard levels. For this purpose, we adopt the two assumptions as follows.

1. The specific humidity on $1000 \mathrm{mb}$ level is conserved horizontally with respect to time.

2. The potential temperature increases linearly with height.

Under the second assumption, the temperature changes on 500 and $700 \mathrm{mb}$ are obtained from those on 600 and $850 \mathrm{mb}$ making use of extrapolation and interpolation method, respectively. Next, with these temperature changes, the upward current $\omega$ is calculated at each standard level except $1000 \mathrm{mb}$. On the other hand, the virtual specific humidity $\Delta t$ later are forecast. In total, therefore, the forecasting of the specific humidity is performed on five levels. Lastly, making use of these elements calculated, we forecast the remaining water vapour on all standard levels.

e) Forecasting Scheme of Precipitation

In this section, we shall summarize the computational process of precipitation. In Fig. 2 , the forecasting procedures are illustrated 


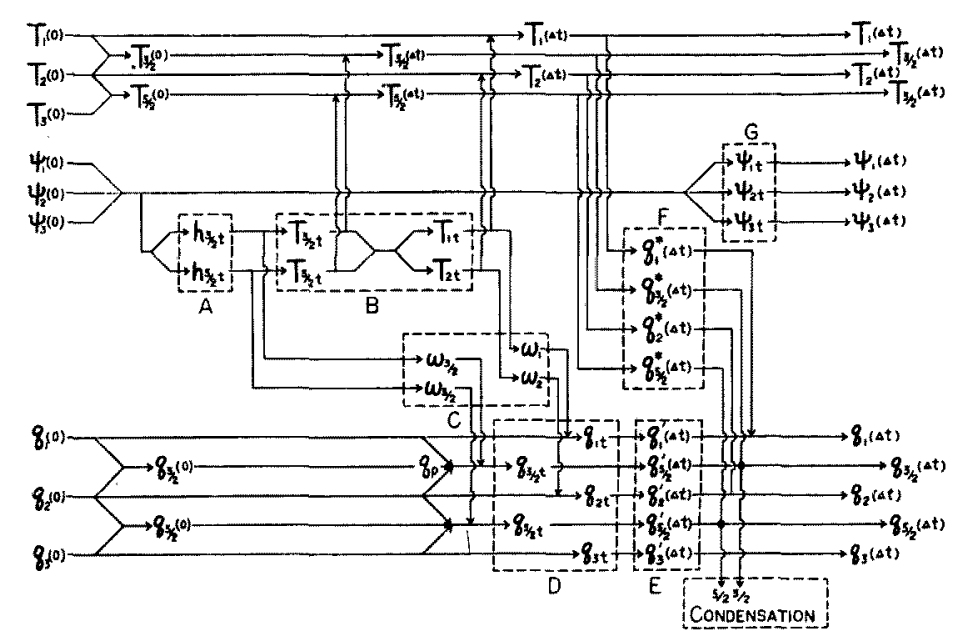

\section{LEGEND \\ NOTATIONS: \\ $T:$ TEMPERATURE \\ $\psi:$ I SOBARIC HEIGHT \\ 8 : SPECIFIC HUMIDITY \\ $8^{*}$ : SAJURATION SPEC. HUMID \\ $q^{\prime}:$ VIRTUAL SPEC. HUMID. \\ $h:$ THICKNESS \\ $w$ : VERTICAL-P-VELOCITY \\ QUANTITIES IN BOXES \\ A: THICKNESS CHANGE \\ B: TEMPERATURE CHANGE \\ $C:$ VERTICAL-P-VELOCITY \\ $D:$ SPECIFIC HUMIDITY CHANGE \\ $E:$ VIRTUAL SPEC. HUMID. \\ $F:$ SATURATION SPEC. HUMID \\ G: HEIGHT CHANGE}

Fig. 2. Flow diagram of rainfall prediction in the case of three layer model. Subscript figures indicate the level numbers shown in Fig. 1, and subscript letters the differential variables: for instance, $T_{1 t}=\frac{\partial T_{1}}{\partial t}$.

schematically in the case of the three layer model. The starting line is at the left of the diagram. At first, the initial data of three meteorological elements, i.e., the heights on isobaric surface, the temperatures and specific humidities on three standard levels are brought to the starting line. Then the calculations are proceeded along the circuits to the direction of arrows indicated. Thus the forecast precipitation is obtained in the lower part of right hand side of the diagram, where "CONDEN. SATION" is marked.

Completion of all the procedures in this diagram means that the calculation for one step is finished, and at the same time, that the calculation for the next step is prepared. In this way we get the amount of precipitation step by step in the course of marching processes.

\section{$\S 3$. Discussion of Results}

In this investigation, we selected the North American Continent for the forecast region. The period for the test was in March. As mentioned above, the scale of the phenomena which is treated herein is so large that we avoided such a season as summer in which small scale convective cells play an important role. On the other hand, in winter, predominant westerlies usually induce large scale upward motions of air in front of a trough, which seems to cause a precipitation over a wide area. Besides, in March, the water vapour content of the air is comparatively high. So, the month was selected for our first trial.

In Fig. 3 the general aspects of the weather condition are shown with the $700 \mathrm{mb}$ chart for 1500 GCT 21 March, 1949, in which we may see a large trough situated in the central part of the United States. On the surface map also, a cyclone corresponding to the trough aloft is located in the middle part. A warm

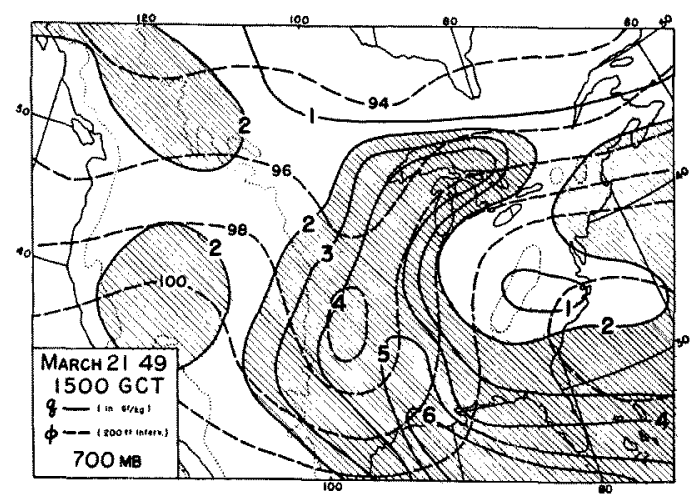

Fig. 3. $700 \mathrm{mb}$ chart for $1500 \mathrm{GCT}$ 21 March 1949 which is used for the calculation as initial pattern, where the dashed lines are height contours and the solid lines the contours of specific humidity. The heights and the water quantities are given in hundreds of feet and $\mathrm{gr} / \mathrm{kg}$, respectively. 


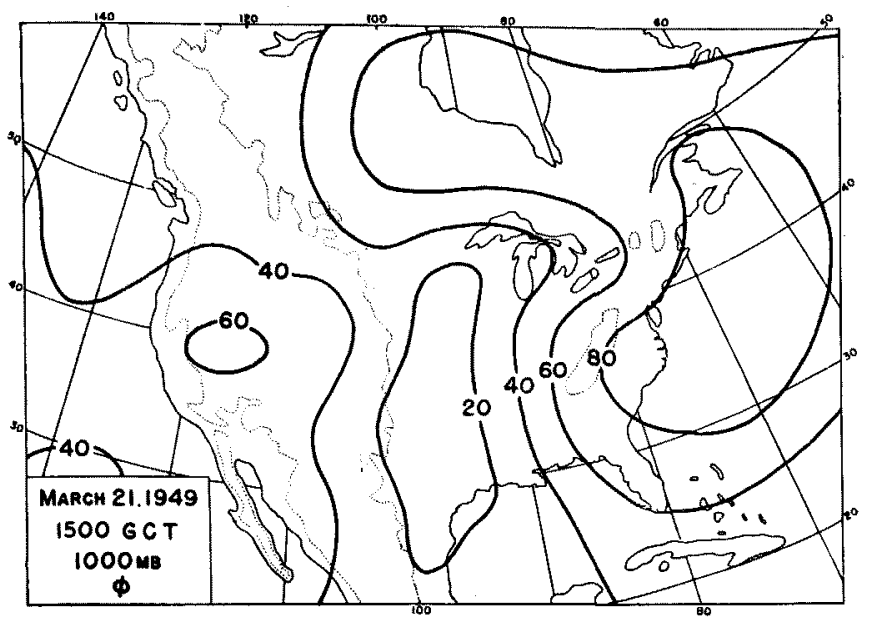

Fig. 4. $1000 \mathrm{mb}$ chart for $1500 \mathrm{GCT} 21$ March 1949 which is used for the calculation as initial pattern, where the solid lines are height contours in the unit of tens of feet.

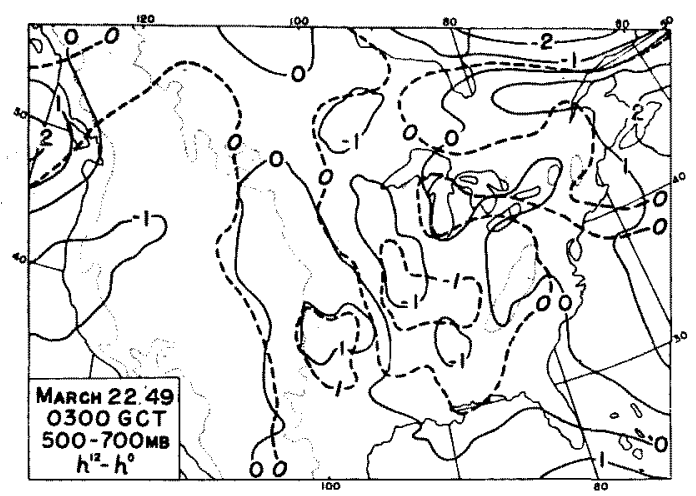

Fig. 5. The observed (solid lines) and forecast (dashed lines) 12 hour thickness changes of the layer between 500 and $700 \mathrm{mb}$ from the ininial time 1500 GCT 21 March 1949. The numbers indicate in hundreds of feet.

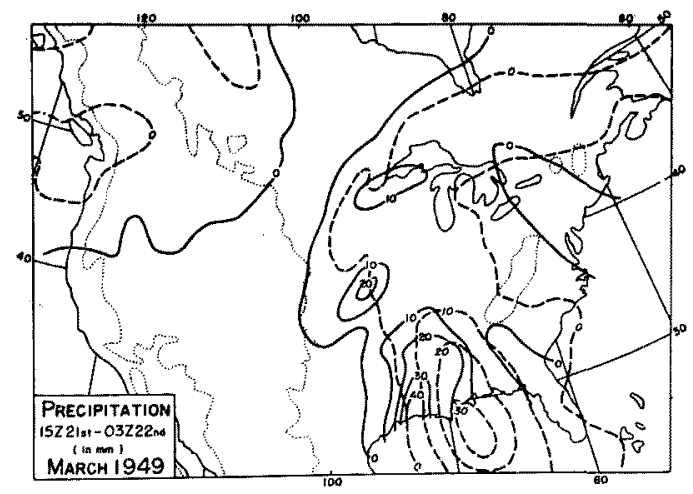

Fig. 6. The observed (solid lines) and the forecast (dashed lines) 12 hour precipitation from 1500 GCT 21 to 0300 GCT 22 March 1949. The contours are drawn in every $10 \mathrm{~mm}$. front extends eastwards from the center of the cyclone to the Great Lakes, and a cold front runs southwestwards to the Pacific Ocean. In the warm section, i.e., in the Greak Lakes region, in New England and in the coastal region of the Gulf of Mexico, much precipitation is observed (Fig. 6). In Fig. 3, the horizontal distribution of water vapour on the $700 \mathrm{mb}$ level is also shown with the specific humidity contours. A moist air tongue as the source of the precipitation is invading from the Gulf of Mexico into the continent.

Next, the result of 12 hour forecast of $500-700 \mathrm{mb}$ thickness change is shown in Fig. 5, which is obtained by the calculation of 2 six-hour time steps. The agreement with the observed pattern is fairly good in each layer over the central and the eastern part of the United States, while some difference existed over and surrounding areas of the Rocky Mountains, especially in the lower layer. This suggests that a topographic effect might have influence upon the change in the thickness pattern. (The correlation coefficients between the observation and the forecast are $0.68(500-700 \mathrm{mb})$ and $0.17(700-1000 \mathrm{mb})$.)

Using the above data, we made 12 hour forecast of precipitation. In Fig. 6 solid lines and dashed lines show the distribution of observed and forecast-amount of precipitation, respectively. So far as the large scale feature

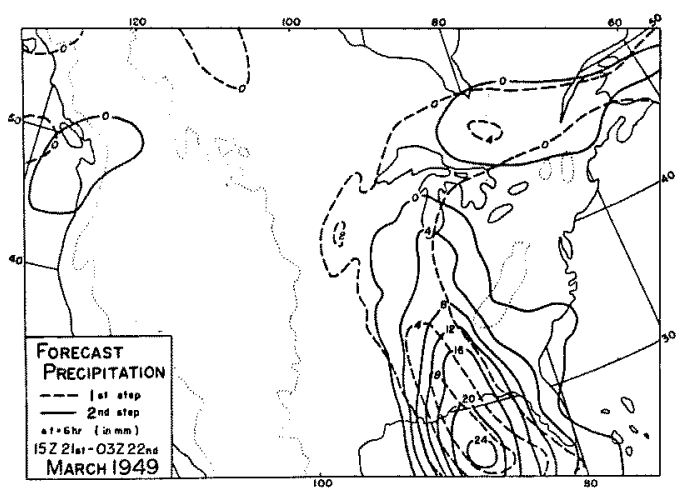

Fig. 7. The first (dashed lines) and the second (solid lines) 6 hour forecast of precipitation from the initial time 1500 GCT 21 March 1949. The amounts are, given in $\mathrm{mm}$. 


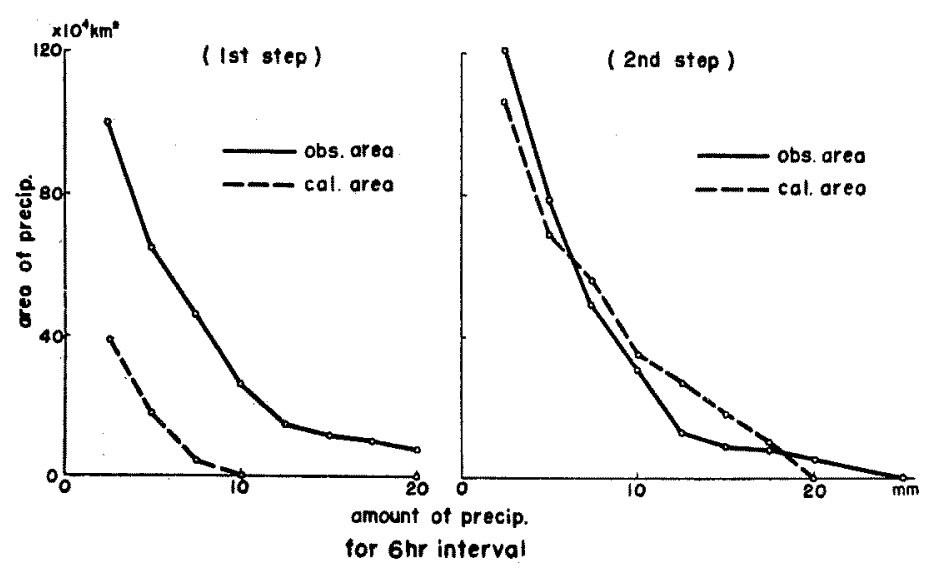

Fig. 8a.

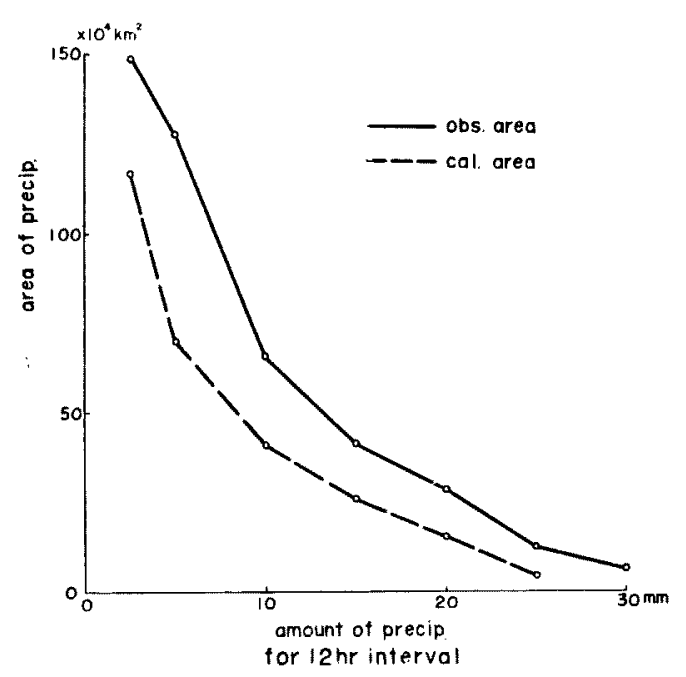

Fig. 8c.

Fig. 8. Precipitation area versus rainfall intensity. Ordinate shows an area where rainfall is larger than stated amount. Solid line and dashed line show the observed and the calculated area, respectively. It is noticed that area shown is confined over the land only, because over the ocean no observation was obtained though forecast was made. a) rainfall in first 6 hours. b) rainfall in second 6 hours. c) total rainfall in 12 hours.

is concerned, these two patterns coincide approximately with each other. However, the forecast pattern was rather flat and dull and the amount of precipitation was generally under-estimated. This under-estimation mainly comes from the first time step interval. In more detail, concerning the observed pattern, the amount of rainfall during the 6 hours is nearly equal to that during the second 6 hours. On the other hand, as shown in
Fig. 7, the forecast amount of precipitation during the first time step interval is one third of that during the second time step. This fact may be more apparent in Fig. 8, where both the forecast and the observed area are shown graphically. In the first 6 hours, the forecast amount is smaller than the observed, but in the next 6 hours, forecast is rather approximate.

The reason may be tentatively explained in the following way. The humidity data by radiosonde has, in general, a tendency to give smaller value than the actual one after the radiosonde comes into cloud. This is proved in Fig. 9, in which contour lines and hatched region show the distribution of relative humidity at $700 \mathrm{mb}$ and the area of the preceeding 6 hour rainfall, respectively. In this figure we can only find very small area where the relative humidity of $100 \%$ is indicated in spite of such a wide rainfall region in reality. Since we used radiosonde data without correction, it may be natural that the amount of forecast rainfall is smaller than the observed at the first step. On the contrary, in the successive steps of the forecasting, residual water vapour content may be conceived to reach $100 \%$ in the portion where oversaturation took place in the previous step. So the shortage of water vapour above mentioned may not appear in

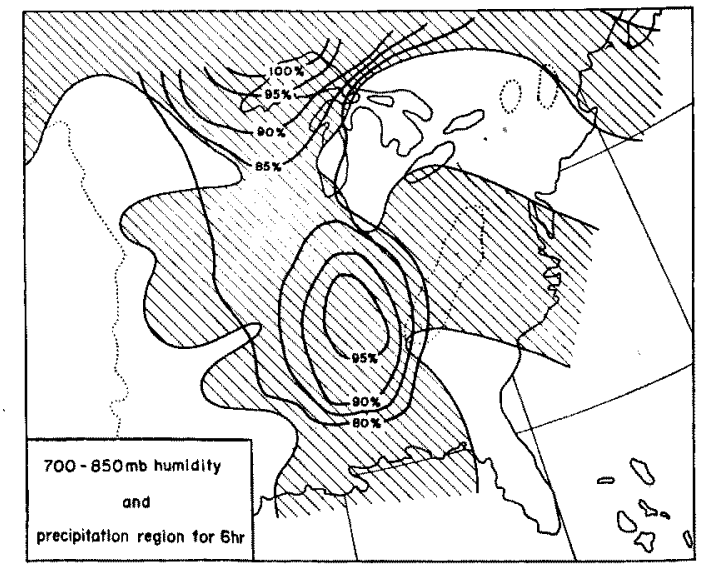

Fig. 9. The mean relative humidity in the layer between 700 and $850 \mathrm{mb}$ on $0300 \mathrm{GCT} 22$ March 1949 (solid lines). The precipitation area of the preceding 6 hours is shaded. 
these steps. In this meaning, we conclude that if the time interval $\Delta t$ is shortened, the more accurate precipitation forecast could be obtained.

\section{§4. Concluding Remarks}

So far, we have performed the numerical prediction of rainfall by the three dimensional pursuit of water vapour and the temperature forecast, and obtained hopeful results for the possibility of the numerical prediction of rain. fall, though they are incomplete. However, there still remain many problems to be solved, which we are going to discuss one by one.

a) The Ground Basis for the Adoption of 3-layer Model.

There are two reasons why we adopt three layer model of the atmosphere. The one is from the vertical distribution of condensation region, and the other from the flow pattern which advects the water vapour.

Concering the former point, we shall quote the result of our preliminary test again. Fig. 10 shows the vertical cross section of condensation distribution along the meridian $90^{\circ} \mathrm{W}$

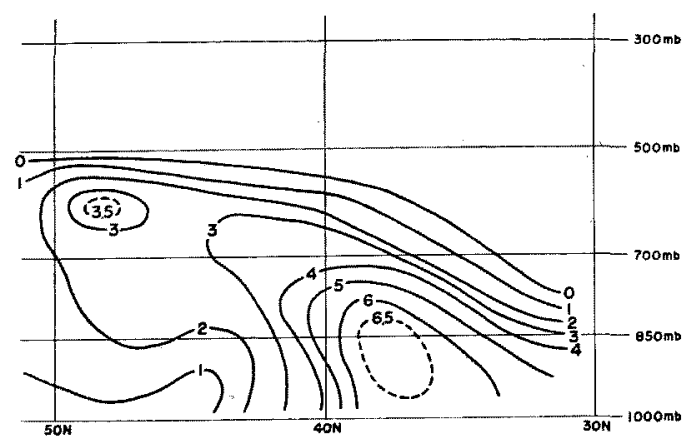

Fig. 10. The vertical distribution of amounts of condensed water vapour for each layer along the meridian $90^{\circ} \mathrm{W}$ from $1500 \mathrm{GCT} 21$ to $0300 \mathrm{GCT} 22$ March 1949. The amounts are given in $\mathrm{gr} / \mathrm{kg}$.

which is obtained dividing the atmosphere into five layers and using the continuity relation for specific humidity of water vapour. This distribution is verified by comparing it with the cloud distribution which is carefully determined by the data of both surface and radiosonde observation. In this figure, we notice two marked centres of condensation regions exist: in the southern part, intense condensation occurs chiefly in the lower layer, i.e.,
$1000-700 \mathrm{mb}$ and in the northern part, it chiefly occurs in the upper layer, i.e., $700-500 \mathrm{mb}$. Therefore, the division of the lower half of the atmosphere into two parts is the least requirement enough to realize the precipitation pattern.

The other reason concerning the advective flow pattern of water vapour is as follows. As is seen Figs. 3 and 4, the wind direction varies rapidly with increasing height in the vicinity of front. Now the air involving water vapour is first advected into the continent from the Gulf of Mexico by the northward flow in the lower layer. Then, it rises up in front of trough, and part of the water vapour contained in it condenses to water drops. The residual water vapour left after the condensation is transported away by the strong westerly current in the upper layer. Therefore, the features of water vapour advection aloft and below are considerably different each other. This fact was also pointed out by Benton and Estoque [4].

\section{b) Effect of Mountain}

As shown in $\$ 3$, the temperature forecast did not result in a success in the mountain region. So, we tried to take into account the topographic effect in the temperature forecast and the calculation of upward current. However, in the existing numerical prediction method, the effect of mountain range is mostly filtered in just the same way as is the small scale patterns and hardly affects the temperature change. As to this problem, Charney and Eliassen [6] made detailed studies previously and obtained the result that the effect of mountain is not so large as might be expected in the height tendency of one day or so. In spite of these results, however, we often observe that the orographic rain is predominant. Especially here in Japan the precipitation of this kind could not be disregarded. Therefore we are forced to solve this problem. One way to overcome this difficulty may be to insert this effect as a second approximation without non-linear coupling with the large scale field and to superpose the effect to the large one.

c) Supply of Water Vapour from the Sea Surface

Another difficult task left for us is the esti- 
mation of water vapour supplied from the sea. Especially, in such a country as Japan surrounded by ocean, the water vapour easily invades into the land from the sea and brings much amount of precipitation. Therefore, it is desirable that the distribution of specific humidity over the ocean is precisely informed. In more detail, two problems should be solved:

1. How to use effectively the sparse data over the ocean?

2. How to estimate the increase in water vapour content of the air traveling over the ocean?

As to the first problem, the similar difficulty also exists in the case of other meteorological elements. In the case of the specific humidity, however, the situation is just different, that is, there is a limiting condition that the relative humidity does not exceed $100 \%$. Hence, provided the temperature distribution is informed, the upper limit of the possible distribution of water vapour can be determined.

Concerning the second problem, Benton and Estoque made labourious and elaborate works, with respect to monthly amount of water vapour over the North American Continent. As seen in Benton and Estoque's studies it seems to be rather important in the comparatively long range forecast to consider the supply of water vapour in the form of evaporation.

d) Application of the Method to the Summer Season

In summer, westerlies in the upper atmosphere are weaker than those in other seasons. Therefore it is expected that the large scale upward current is also weak and the effect of the small scale variation of vertical static stability may predominate. It is an interesting task to apply our method directly to such weather situation and to discuss the discrepancy to be found.

\section{e) Non-adiabatic Effect}

It is a remarkable fact that the useful result for the forecast of precipitation was obtained by the temperature forecast in spite of the adiabatic assumption we adopted. However, it may be too hasty to draw a decisive conclusion from the present example that nonadiabatic effect does not contribute to the change in thickness pattern. As is seen from
Fig. 10 which shows the vertical distribution of condensation, the average change of specific humidity between $600 \mathrm{mb}$ and $1000 \mathrm{mb}$ is about $2.5 \mathrm{gr} / \mathrm{kg} / 12 \mathrm{hr}$ in the region of intense rainfall. If we estimate the corresponding temperature increase due to the released heat assuming static thermal expansion, it amounts to as much as $5.6^{\circ} \mathrm{C} / 12 \mathrm{hr}$, which exceeds by far the maximum of the adiabatic temperature change $\left(4^{\circ} \mathrm{C}\right)$. So there must be some mechanism which diminished the above temperature change so that our adiabatic assumption is plausible.

Here, in this section, we shall quote the results of model research performed by one of the authors, Manabe [13], concerning the contribution of heat released by condensation to the change of thickness pattern, i.e., the change of large scale temperature field. Now, instead of potential temperature, equivalent potential temperature is conserved when condensation takes place. Accordingly,

$\frac{d}{d t} \ln \theta_{e}=\frac{D}{D t} \ln \theta+\omega \frac{\partial \ln \theta}{\partial p}+\frac{L_{i} \times 10^{-3}}{C_{p}} \frac{d}{d t}\left(\frac{q}{T}\right)=0$

or

$$
\omega \fallingdotseq \kappa \delta p\left(\frac{D}{D t} \ln \theta+\frac{L_{t} \times 10^{-3}}{C_{p} T} \frac{d q}{d t}\right)
$$

where $\theta_{e}=\theta \exp \left(L_{t} / C_{p} T\right)$ is equivalent potential temperature, $L_{t}$ latent heat of condensation, $C_{p}$ specific heat at constant pressure. Inserting the above equation (4.2) into the vorticity equation (2.1), and transforming this in the same way as in $\$ 2$, we can obtain the following equation corresponding to equation (2.8):

$$
\frac{D_{\tau}}{D t}=\frac{\partial \boldsymbol{V}}{\partial p} \cdot \delta p \cdot \nabla \xi-\kappa \delta p f^{2} \frac{L_{t}}{C_{p} T} \frac{\partial^{2}}{\partial p^{2}}\left(\frac{d q}{d t}\right) .
$$

In order to restrict our discussion to the problem of non-adiabatic effect, we devided $\tau$, into two parts, i.e.,

$$
\tau_{t}=\tau_{t}^{a}+\tau_{t}^{n a}
$$

where

$$
\tau_{t}^{n a}=-\kappa \delta p f^{2} \frac{L_{t}}{C_{p} \bar{T}} \frac{\partial^{2}}{\partial p^{2}}\left(\frac{d q}{d t}\right)
$$

Such separation, of course, holds only for the first time step, for at initial time $V^{n a}=0$ and $\eta^{n a}=0$ are assumed. After second step, it is difficult to distinguish them on account of the complicated non-linear coupling. Therefore, 
present discussion is confined to the initial tendency.

From the above equation (4.4), $\tau_{l}^{n a}$ and accordingly $h^{n n}$ is to be calculated if the initial distribution of condensation is given.

In Fig. 11, the horizontal distribution of condensation is shown. As for the vertical direction, the distribution is assumed to be of the discrete form: $d q / d t$ is maximum at 850 $\mathrm{mb}$ level and zero at $1000 \mathrm{mb}$ and $450 \mathrm{mb}$.

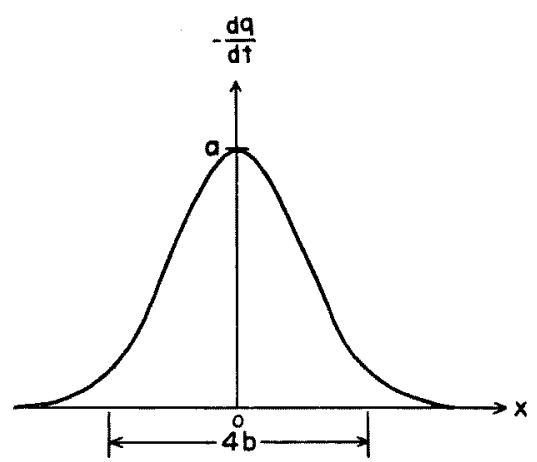

Fig. 11. Horizontal distribution (Gaussian distribution) of condensation - $d q / d t$ used for model research. $x$-axis points positive eastward and $d q / d t$ is assumed to be independent of latitude. $b$ is the standard deviation of this distribution and $4 b$ roughly corresponds to the representative east-westward scale of the precipitation region.

Expressing this in mathematical form,

$$
\begin{aligned}
\frac{d q}{d t}= & -\exp \left[-\frac{x^{2}}{2 b}\right] \text { (per } 12 \mathrm{hr} \text { ) between } 1000 \mathrm{mb} \\
& \text { and } 600 \mathrm{mb} \\
=0 & \text { at } 1000 \mathrm{mb} \text { and above } 450 \mathrm{mb}
\end{aligned}
$$

where $x$ is the horizontal coordinate pointing eastward and $b$ is the standard deviation of horizontal extension of this distribution, which represents the measure of width of condensation area. Using the above functional form of distribution, the initial non-adiabatic thickness tendency between $1000 \mathrm{mb}-600 \mathrm{mb}$ is approximately computed as follows,

$$
\begin{aligned}
\partial h_{10 \sim 0^{n a}} & =\frac{1}{\sqrt{2}} \bar{\mu} b e^{\frac{P^{2} b^{2}}{2}}\left(e^{-v^{2} P x} \int_{\lambda}^{\infty} e^{v^{2}} d v\right. \\
& \left.+e^{P x} \int_{\nu}^{\infty} e^{-v^{2}} d v\right)
\end{aligned}
$$

where

$$
\lambda=\frac{P b}{\sqrt{2}}-\frac{x}{\sqrt{2 b}}, \quad \nu=\frac{P b}{\sqrt{2}}+\frac{x}{\sqrt{2 b}}
$$

and $\bar{\mu}$ and $P$ are constants depending on static stability and the thickness of the layer ${ }^{4)}$.

Fig. 12 shows the relation between the magnitude of non-adiabatic effect and the

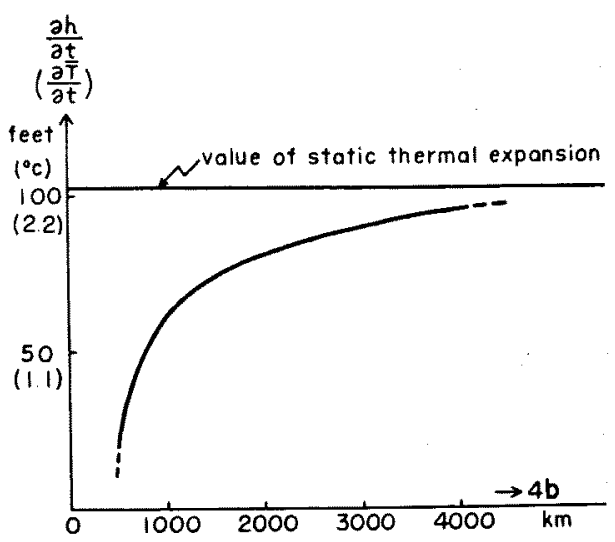

Fig. 12. The relation between the horizontal scale of condensation and the initial tendency of non-adiabatic thickness change. In ordinate, nonadiabatic part of thickness change (feet/12 hr) for the layer between 1000 and $600 \mathrm{mb}$ levels at the center of precipitation area is taken, and, in abscissa, the representative value of the scale of the area.

scale of horizontal extension of rainfall which is calculated from the above solution (4.6). Here, in ordinate, non-adiabatic part of thickness change at the centre of precipitation area is taken, and, in abscissa, the representative value of the scale of the area. This figure suggests that the narrower the precipitation region is, the smaller the thickness change and only when $b \rightarrow \infty$, thickness change tends to the value evaluated from the assumption of mere thermal expansion of air column.

On the other hand, in the case of extremely small scale source region, the present results derived under the geostrophic approximation is not applicable. Simple consideration leads, however, to the conclusion that the case $b=0$ corresponds to the one treated by "Parcel Method ". It is obvious that in this treatment the temperature at a fixed point is substantially unchanged, because it is assumed that the heated air parcel rising upward stops at the level where the temperature of surrounding atmosphere equals to that of air parcel. There-

4) Here, as the boundary conditions, $\omega^{n a}=0$ at $p=0$ and $p=1000 \mathrm{mb}$ and $h_{t}^{n a}=0$ at $x= \pm \infty$. 
fore the curve in Fig. 12 is supposed to tend to zero when $b \rightarrow 0$.

From the above results it is concluded that the non-adiabatic temperature change is considerably smaller than the value estimated from the assumption of the static thermal ex. pansion. Besides, the effect of radiation acts in the opposite sense to that of condensation. Especially long wave radiation cools cloud appreciably. These are reasons why we could obtain the useful temperature forecast for the forecast of precipitation with the adiabatic assumption. Therefore, both the result of precipitation forecast and the discussion of non-adiabatic effect in this section suggests the fact that large scale precipitation pattern is mostly explained by the vertical velocity calculated under the adiabatic assumption, and in this season, spring, the small scale convection perhaps play a secondary role in the precipitation mechanism.

\section{Acknowledgements}

The authors express their cordial thanks to Prof. S. Syono, who has always encouraged them, to Prof. K. Isono, Dr. Y. Ogura and Dr. K. Gambo, who have given them many effective suggestions. They are indebted to Mr. K. Mohri, Central Meteorological Observatory, who kindly revised the maps for forecasts and to Mr. H. Tani, CMO, who prepared the data necessary for their investigation. They also wish to thank Mr. Y. Fujiki for his labourious work of tracing the figures.

\section{References}

1. Arizumi, N. (1949): On the Graphical Computing Method of Vertical Current and the Forecasting of Cyclone Track by this Method. Journ. Meteor. Res. 1, 71-79. (in Japanese)

2- Asakura, T. (1953): Forecasting of Precipitation. Journ. Met. Soc. Japan 31, 306-312. (in Japanese)

3. Bellamy, J. C. (1949): Objective Calculations of Divergence, Vertical Velocity and Vorticity. Bull. Amer. Meteor. Soc. 30, 45-49.

4. Benton, G. S. and Estoque, M. A. (1954): Water-vapour Transfer over the North American Continent. Journ. Meteor. 11, 462-477.

5. Charney, J. G. (1954): Numerical Prediction of Cyclogenesis. Proc. Nat. Acad. of Sciences 40, 99-110.

6. Charney, J. G. and Eliassen, A. (1949): A
Numerica 1 Method for Predicting the Perturbations of the Middle Latitude Westerlies. Tellus 1, No. 2, 38-54.

7. Charney, J. G. and Phillips, N. A. (1953): Numerical Integration of the Quasi-geostrophic Equation for Barotropic and Simple Baroclinic Flows. Journ. Meteor. 10, 71-99.

8. Collins, G. O. and Kuhn, P. M. (1954): A Generalized Study of Precipitation Forecasting. Part 3: Computation of Precipitation resulting from Vertical Velocities deduced from Vorticity Changes. Mon. Wea. Rev. 82, 173-182.

9. Eliassen, A. and Hubert, W. E. (1953): Computations of Vertical Motion and Vorticity Budget in a Blocking Situation. Tellus 5 . 196-206.

10. Fjфrtoft, R. (1952): On Numerical Method of Integrating the Barotropic Vorticity Equation. Tellus 4, 179-194.

11. Fliegle, J. C. (1946): A Study of the Effects of Divergence and Advection on Lapse Rate. Journ. Metecr. 3, 9-13.

12. Kuhn, P. M. (1953): A Generalized Study of Precipitation Forecasting. Part 2: A Graphical Computation of Precipitation. Mon Wea. Rev. 81, 381-394.

13. Manabe, S. (1955): On the Contribution of Heat relased by Condensation to the Change in Pressure Pattern. (to be published)

14. Masuda, Y. (1950): On the Method of Computing Vertical Motion in the Atmosphere. Pap. Met. Geophys. 1, 1-8, 9-19, 20-28.

15. Miller, J. E., (1946): Application of Vertical Velocities to Objective Weather Forecasting. Prog. Rep., Mimeogr., College of Engineering, New York University.

16. Panofsky, H. A. (1952): Large-scale Vertical Velocity and Divergence. Compendium of Meteorology, Amer. Meteor. Soc., Boston. 639646.

17. Phillips, N. A. (1951): A Simple Three-dimensional Model for the Study of Large-scale Extratropical Flow Pattern. Journ. Meteor. $8,381-394$.

18. Spar, J. (1953): A Suggested Technique for Quantitative Precipitation Forecasting. Mon. Wea. Rev. 81, 217-221.

19. Syōno, S. (1950): On the Vortical Rain. Geo. phys. Notes, Tokyo Univ. 3, No. 25.

20. Thompson, J. C. and Collins, G. O. (1953): A Generalized Study of Precipitation Forecasting. Part 1: Computation of Precipitation from the Fields of Moisture and Wind. Mon. Wea. Rev. 81, 91-100.

\section{Sources of Data}

Daily Series Synoptic Weather Maps. March 1949. U.S. Weather Bureau.

Daily Upper Air Bulletin. March 1949. U.S. Weather Bureau.

U.S.A. Climatological Data. Murch 1949, U.S. Weather Bureau, 